\title{
Active Contours Driven by Cellular Neural Networks for Image Segmentation in Biomedical Applications
}

\author{
Bogdan BELEAN \\ National Institute for Research and Development of Isotopic and Molecular Technologies, \\ Centre of Research and Advanced Technologies for Alternative Energies, Cluj-Napoca, Romania \\ bogdan.belean@itim-cj.ro
}

\begin{abstract}
This paper proposes a novel approach for image segmentation in the context of biomedical applications. The medical images considered for this analysis are both microarray images and images recorded from microfluidics devices. In case of microarray images, microarray spots represented as circular shapes are localised and used further on for the estimation of gene expression levels, based on the average pixel intensities. Considering the microfluidic devices images, the features of cells or clusters of cells are determined by using the proposed image segmentation approach. The novelty of this approach lies in the fact that this is a segmentation procedure which uses an edge-based active contour model (ACM) driven by cellular neural networks (CNNs). Thus, a predefined curve is evolved towards the edges of the image objects (i.e., circular microarray spots and irregular shapes representing clusters of cells). In the curve evolution process, the classic representation of image edges by using the gradient vector is replaced by an edge-based feature template determined by the CNN for each image object. The benefits of the proposed segmentation method are illustrated for both image processing applications, by using specific quality measures for the characterization of object features within the image under analysis.
\end{abstract}

Keywords: Image segmentation, Active contours, Cellular neural networks, Microarray, Microfluidics, Gene expression, Cell features, Cell clusters.

\section{Introduction}

Image segmentation is a key area of computer vision that has gained a lot of research attention in recent years. The aim of image segmentation is to divide an image into multiple segments with similar features such as texture, strength or color for examining objects within the image under analysis. Image segmentation has numerous applications in industry and research, including image or object recognition, image processing and comprehension, medical diagnosis of diseases, computer-guided surgery, treatment planning and anatomical structure. As it is presented in (Pal \& Pal, 1993), conventional image segmentation techniques can be classified as threshold-based segmentation (Vese \& Guyader, 2015), clustering-based segmentation (Wang \& Huang, 2009; Hrošik et al., 2019), segmentation based on neural networks and region-based segmentation. Considering the last category, active contour models - ACM (Zou et al., 2021) are defined as an evolving contour towards the object boundaries. The curve evolution is led by the minimization of an energy functional with different terms such as membrane term causing the curve to shrink, the rigidity term which encourages the border to be a straight line and last but not least the term which pushes the curve to the object contour. Classic approaches, such as the edge-based models use the image gradient information to define object boundaries and stop the contour from evolving. In the presence of weak and noisy edges, the precision of the approaches where gradient information was used to direct an evolving contour toward the object boundaries is diminished. This method is sensitive to the initial level set position and the level set can easily break through the edges which are weakly expressed (Rahmat \& HarrisBirtill, 2018). Active contour models, in which an energy mechanism is formulated based on information such as strength, color, or texture in order to evolve an initial contour towards the object boundaries (Han, Zhang, \& Gao, 2018), provide improved segmentation accuracy. The aforementioned approaches which are known as region-based active contours, show increased performance on weak boundaries and they are usually less sensitive to noises and initial contours. Considering the region-based basic assumption that each segmented area has constant intensity, these approaches perform poorly on images with intensity inhomogeneity. To overcome these limitations, the present paper proposes a cellular neural network (CNN) approach for edge features determination, where intensity inhomogeneity is accounted for and also the weakly expressed edges are enhanced. This is mainly due to the determination of an adaptive edge features template that is embedded within the curve evolution process. Results are illustrated in the case of two biomedical applications, microarray imaging for gene 
expression levels estimation and microfluidic devices for cell clusters features determination. Thus, the remainder of this paper is structured as follows. The basic principle of edge-based ACM together with the CNN approach for edge features template computation are presented in Section 2. In Section 3, the proposed CNNdriven active contour approach is employed in the case of biomedical imaging applications. The fourth section presents the conclusions of this paper.

\section{Method Description}

The proposed approach determines the edge features using an unsupervised machine learning approach. The detected features are further on used in a curve evolution procedure in order to determine pixels corresponding to the image foreground (i.e., the objects within the image which are cells, cell clusters or microarray spots) and its background. In this way, image segmentation is performed by separating foreground pixels from the image background by evolving an initial curve toward the object boundaries. The behavior of the proposed image processing workflow for segmentation is exemplified by means of an image with two cells used in (Li et al., 2010) and another image with cells registered using microfluidic devices (see Figures 2 and 3). To sum up, there are two main steps of the proposed segmentation procedure: cellular neural networks for edge features computation and active contours for the segmentation of image objects.

\subsection{Cellular Neural Networks - CNN}

There are both supervised and unsupervised machine learning approaches for image segmentation available. The accuracy of supervised ML-based segmentation is improved, since the input data is labeled and well-known (Xing et al., 2018; Hagerty et al., 2019). Unsupervised learning, on the other hand, has the benefit of automatic segmentation without any previofus knowledge of the object features in the training dataset (Jiao, Chen \& Dong, 2020), albeit at the cost of increased computational complexity (Daniels \& Gallagher, 2018). This is also the case of the described approach, namely edge features detection using cellular neural networks. The main benefit is that no training sessions are needed, and edge features are enhanced in comparison with the gradientbased edge detection approaches. Cellular neural networks (CNN) were introduced in 1988 and 1993 (Chua \& Yang, 1988; Roska \& Vanderwalle, 1993). Arrays of similar dynamical structures, known as cells, are connected locally in such networks (Roska \& Vanderwalle, 1993). The basic unit of a CNN is the cell, which is a one-dimensional dynamic structure connected only to its neighbors, allowing neighboring cells to interact directly (Figure 1(a)). The $\mathrm{CNN}$ dynamics is described by the nonlinear differential equation of a $C_{i j}$ cell in position $(i, j)$ with uij, xij, yij the input, the state and the output of the aforementioned $C_{i j}$ cell as follows:

$$
\begin{aligned}
& \frac{d x_{i j}(t)}{d t}=-x_{i j}(t)+\sum_{(k, l) \in N_{i j}} A(i, j ; k, l) y_{k l}(t) \\
& +\sum_{(k, l) \in N_{i j}} B(i, j ; k, l) u_{k l}(t)+z(i, j ; k, l)
\end{aligned}
$$

where the indices $k$ and $l$ denote a given cell belonging to the neighborhood of the $C_{i j}$ cell. The neighbours of the $C_{i j}$ cell are denoted by $N_{i j}^{r}$ and defined as

$$
N_{i j}{ }^{r}=\left\{\begin{array}{l}
x_{k l} \mid \max \{|k-i|,|l-j|\} \leq r, \\
1 \leq k \leq M, 1 \leq l \leq N
\end{array}\right\}
$$

where $M$ and $N$ represent the size of the twodimensional array of cells represented in Figure 1(a).

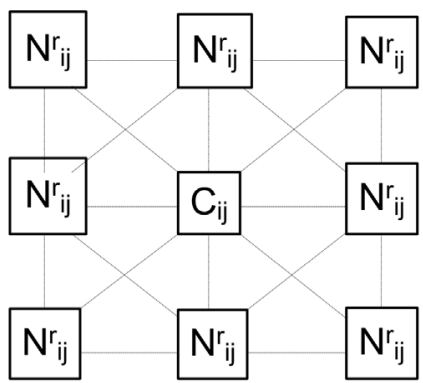

a)

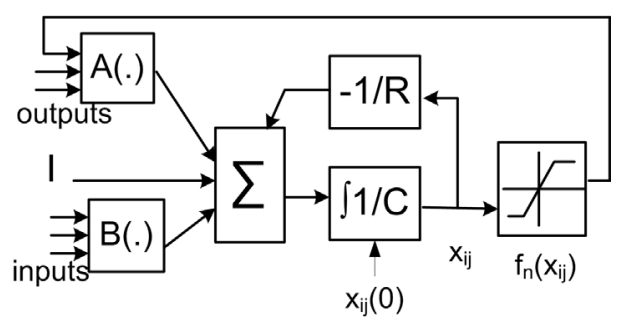

b)

Figure 1. (a) Interconnection of $M \mathrm{x} N$ two-dimensional block of cells $\left(C_{i j}\right.$ cell and its neughbours $N^{r}$ ) within the CNN approach (Roska \& Vanderwalle, 1993); (b) $\mathrm{CNN}$ cell state computation based on the template matrices and the configuration constants 
A constant external input $u$ and an output $y$ are two main characteristics of each neural network cell. The block diagram for the computation of each neural network cell state is shown in Figure 1(b). It includes linear and nonlinear elements that serve a given purpose according to their configuration constants $x_{i j}(0)$, R and $\left.\mathrm{C}\left|x_{i j}(0)\right| \leq 1, \mathrm{C}>0, \mathrm{R}>0\right)$ and to the template matrices $\mathrm{A}($.$) and \mathrm{B}($.$) , where$ $\mathrm{A}($.$) affects the output control of the neighboring$ cells and it is known as the feedback operator, whereas $\mathrm{B}($.$) affects the input control and is$ known as the control operator. Each element of the template matrices corresponds to a cell from the CNN structure. A two-dimensional CNN can be thought of as a parallel non-linear twodimensional filter that can be used for various tasks. In image processing, noise removal ( $\mathrm{Su}$ et al., 2008), shape extraction, edge detection, and inpainting (Elango \& Murugesan, 2009) can be achieved by calibrating the A, B, and I template coefficients. According to (Li et al., 2011; Nossek, 1996), the determination of template coefficients is performed either by design or by learning. In the present case, for extracting the image edges and simultaneously suppressing the noise, solving the system of inequalities as it is described in (Nossek, 1996) led to the following template matrices:

$$
\begin{aligned}
A & =\left(\begin{array}{ccccc}
-1.29 & 2.58 & -1.29 & 2.58 & -1.29 \\
2.58 & 0.83 & 1.58 & 0.83 & 2.58 \\
-1.29 & 1.58 & 8.67 & 1.58 & -1.29 \\
2.58 & 0.83 & 1.58 & 0.83 & 2.58 \\
-1.29 & 2.58 & -1.29 & 2.58 & -1.29
\end{array}\right), \\
B & =\left(\begin{array}{ccccc}
0 & -3 & -1 & -3 & 0 \\
-3 & 2 & 3 & 2 & -3 \\
-1 & 3 & 8 & 3 & -1 \\
-3 & 2 & 3 & 2 & -3 \\
0 & -3 & -1 & -3 & 0
\end{array}\right), I=-1 .
\end{aligned}
$$

In the case of biomedical images, the values of pixel intensities vary significantly in the areas corresponding to object edges. These phenomena lead to unexpected results when noisy images are processed by means of classical edge detection operators, such as Roberts, Sobel, Prewitt. Because of inhomogeneous foreground, background and noisy edges, it is unrealistic to find a uniform threshold suitable for every image object. Thus, the proposed CNN-based edge features detection is sensitive to both strong and weak edges. As an example, in Figure 2 where edges are strongly expressed, the active contour procedure behaves similarly when it is based on gradient matrix (Figure 2(a)) and based on edge features determined by CNN (Figure 2(b)).
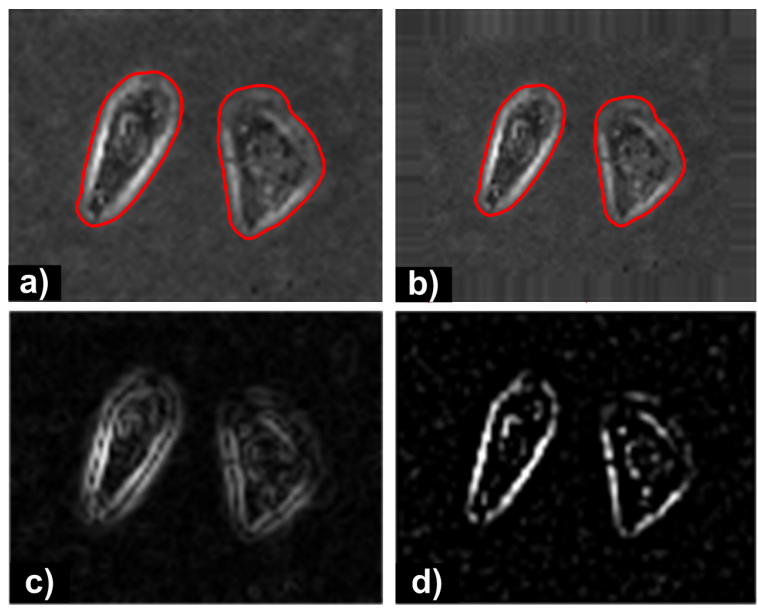

Figure 2. (a) Image of two cells from (Li et al., 2010) with the evolved curve based on gradient information around the object boundaries; (b) Image of two cells with evolved curve based on edge features determined by CNN; (c) Gradient magnitude representation; (d) Edge features determined by $\mathrm{CNN}$

On the other hand, in the case of weakly expressed edges, active contours driven by CNN edge features (Figure 3(d)) offer better segmentation results than the classic active contour based on the magnitude of the image gradient (Figure 3(c)). Thus, it can be seen in Figure 3(b) how the lower right part of the evolved edge described the cell more accurately than the one from Figure 3(a).

To better understand the way a CNN works, it can be seen as an iterative spatial convolution process on bi-dimensional matrices corresponding to image pixel intensity values. At the price of increased computational cost, the edges are detected and enhanced, a benefit that can be used in cases where it is difficult to distinguish the useful information from he background. As an example, X-ray images are processed for edge detection (Prasad et al., 2016). Nevertheless, the proposed approach is not limited to bidimensional filtering. It also can be employed in processing uni-dimensional image profiles for the determination of the magnitude and location of profile peaks. As a result, increased accuracy for the determination of structural ordering parameters is obtained (Gavrea et al., 2017). Further on, the procedure of evolving an initial contour based on edge features determined by $\mathrm{CNN}$ is explained. 

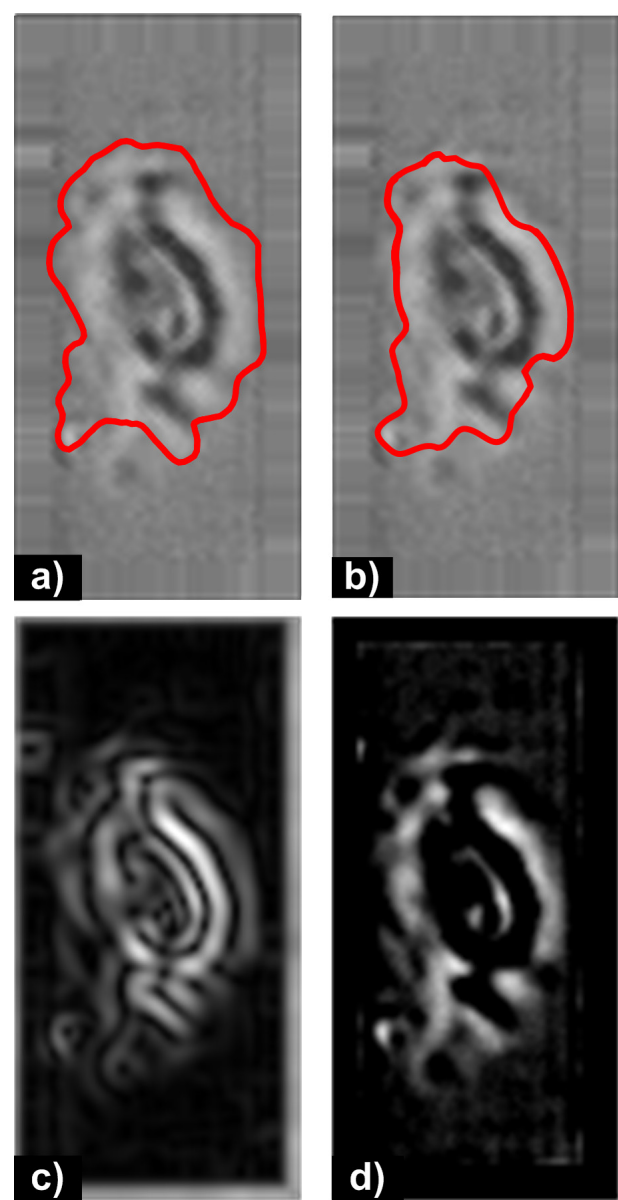

Figure 3. (a) Image of a microfluidics cell with the evolved curved based on gradient information around the object boundaries; (b) Image of a microfluidics cell with the evolved curved based on edge features determined by $\mathrm{CNN}$; (c) Gradient magnitude representation; (d) Edge features determined by $\mathrm{CNN}$

For this purpose, equation (2) and the $A($.$) ,$ $B($.$) and I are used for the determination of the$ edge features denoted by the $g^{C N N}{ }_{i j}$ values which correspond to the states of $C_{i j}$ cells.

$C \frac{d g_{i j}{ }^{C N N}(t)}{d t}=$

$-\frac{1}{R} g_{i j}^{C N N}(t) \sum_{C_{k l} \in N^{r}{ }_{i j}} A(i, j ; k, l) y_{k l}(t)+$

$\sum_{C_{k l} \in N^{r}} B(i, j ; k, l) u_{k l}(t)$

where:

- $\quad C$ and $R$ are integration constants;

- $\quad I$ is an independent bias constant;

- $\quad y_{i j}(t)=f\left(g_{i j}{ }^{C N N}(t)\right)$

- $\quad f(x)=\frac{1}{2}(|x+1|-|x-1|)$.

\subsection{Active Contour Models Driven by CNN}

The active contour procedure for image segmentation is presented as follows. Let $I S$ be an image defined on a given domain $\Omega$, containing the background pixels and the foreground pixels associated with the image objects. One considers an initial rectangular perimeter that confines both background and foreground pixels. The aforementioned perimeter is evolved towards the boundaries of the objects within the image under analysis. In order to describe the curve evolution process, a given dynamic contour is defined as the zero level set of a time dependent energy functional $\operatorname{LSF} \varphi(x, y, t)$. Assuming that the LSF $\varphi$ takes negative values inside the zero level contour and positivie values outside, in order to determine the boundaries of the objects, one has to find the solution of the partial differential equation denoted by (3), which is referred to as the level set evolution (Li et al., 2010):

$\frac{\partial \varphi}{\partial t}=F|\nabla \varphi|$

where $\mathrm{F}$ is the speed function that controls the motion of the curve.

The zero level of the level set function (LSF) $\varphi(x, y, t)$ corresponds to the object boundaries. The $\operatorname{LSF} \varphi$ must be smooth and accurate in image segmentation applications, particularly near its zero-level set, where it describes the contour of the object to be determined. In order for the level set to be applied for the determination of object boundaries, according to (Li et al., 2010), the previous equation is written as:

$\frac{\partial \varphi}{\partial t}=\operatorname{div}\left(\frac{|\nabla \varphi|-1}{|\nabla \varphi|} \nabla \varphi\right)-\lambda \frac{\partial e_{e x t}}{\partial \varphi}$,

where $e_{e x t}$ is chosen to describe the edge information. The classic approach makes use of the following $L$ and $A$ energy functionals: $e_{e x t}=\lambda L(\varphi)+\alpha A(\varphi)$,

with

$$
\begin{aligned}
& L(\varphi)=\int_{\Omega} g \delta(\varphi)|\nabla \varphi| d x, \\
& A(\varphi)=\int_{\Omega} g H(-\varphi) d x,
\end{aligned}
$$

where the coefficient $\lambda>0, \alpha \in \mathfrak{R}, \delta$ is the Dirac delta function and $H$ the Heaviside function. The $A$ term speeds up the curve motion in case the initial contour is far away from the desired boundaries, 
whereas the $L$ term computes the line integral of the function $g$ along the zero level contour of $\varphi$, pointing to a minimum in case $\varphi$ is located at the object boundaries. The function $g$ represents the edge indicator function and it is defined as:

$$
g=\frac{1}{1+\left|\nabla G_{\sigma} * I_{S}\right|^{2}},
$$

where $G_{\sigma}$ is a Gaussian kernel with a standard deviation $\sigma$ used to smooth the Is image by using the convolution operator *.

The results of the segmentation performed by means of active contours based on the edge indicator function expressed as equation (5) can be seen in Figure 2(a) and Figure 3(a) in the case of two distinct types of cells.

By replacing the edge information function $g$ with the $g^{C N N}$ from equation (2), equation (4) is transformed as follows:

$$
\begin{aligned}
& \frac{\partial \varphi}{\partial t}=\operatorname{div}\left(\frac{|\nabla \varphi|-1}{|\nabla \varphi|} \nabla \varphi\right)- \\
& \lambda \delta(\varphi) \operatorname{div}\left(g^{C N N} \frac{\nabla \varphi}{|\nabla \varphi|}\right)-\alpha g^{C N N} g \delta(\varphi)
\end{aligned}
$$

where $\delta$ represents the smooth approximations of the Dirac delta function. An improvement of the segmentation procedure can be observed in the case of weakly expressed edges like the ones from Figure 3(b) in comparison with Figure 3(a).

The proposed segmentation procedure is applied to two types of biomedical images, namely microarray images and images recorded from microfluidics devices. The results of the segmentation procedure based on edge information delivered by the $\mathrm{CNN}$ approach are presented in the next section. Pre-processing techniques can be added for contrast enhancement in case the application demands it. Future work is aimed at such implementations, considering the computationally efficient techniques for local contrast analysis available for the improvement of the image segmentation process (Vrejoiu, 2020). In order to reduce the computational time needed for the curve to evolve towards its final state which corresponds to object boundaries, selective diffusion as it is proposed in (Terebes et al., 2018) can be also applied. Moreover, object texture (Barburiceanu, Terebes \& Meza, 2021) can be also embedded within the curve evolution process in a similar manner as the proposed approach. This shows once again that the active contour model remains an important tool for image segmentation.

\section{Experimental Results}

\subsection{Microarray Image Analysis}

Microarray images are used for the estimation of gene expression levels. RNA derived from biological samples is synthesized into microarray targets in microarray experiments. Singlestranded DNAs or RNAs representing individual genes, labeled with fluorescent markers, are the targets. The microarray targets hybridize with sub-sequences (probes) of the genes within the whole genome on a microarray slide, with each gene paired with a fluorescent spot. For each fluorescent marker, a .tiff image is created using laser scanning at the appropriate wavelengths. In a two-color microarray experiment, a probe image, which corresponds to one fluorescent label, is compared to a reference image, which corresponds to the other fluorescent label. Using advanced microarray image processing techniques, gene expression levels are determined based on fluorescent light intensities (Campbell, Hatfield \& Heyer, 2007). Microarray technology is intensively used in describing biological mechanisms involved in various diseases (Venkataramana et al., 2018; Belean et al., 2015).

The selection of the rectangular region for each spot using the method proposed in (Belean et al., 2020) is the first step in segmenting microarray spots using active contours. Both the background and foreground pixels associated with the microarray spot are included in the rectangular region. The foreground pixels correspond to microarray spot pixels, while the background pixels correspond to the local spot background. As a result, the second step is to divide pixels within each rectangular region into foreground and background. For each rectangular region, the proposed level set approach guided by the CNN edge features is employed in order to determine the contour of the microarray spot. The dataset onto which the proposed method is applied is presented next.

\subsection{Dataset, results and discussion}

One used 4 pairs of images corresponding to the microarray samples having the following IDs: GSM333336, GSM333353, GSM333337 and GSM333341 (according to the microarray analysis conducted by (Fabro et al., 2008) listed in Gene Expression Omnibus). Each pair consists 
of two images corresponding to the $\mathrm{I}_{\text {cy3 }}$ and $\mathrm{I}_{\mathrm{cy} 5}$ fluorescent dyes.

Each microarray image has a size of 4000x1944 pixels and contains 32 spot groups with 380 spots per group. The GSM333336 and GSM333353 samples represent biological replicates of uninfected Arabidopsis leaves compared to a common reference and they are further on reffered to as E1 samples. The GSM333337 and GSM333341 samples are denoted by $E 2$ and they represent biological replicates of infected leaves compared to the same reference. A selection of microarray spots is used for illustrating the visual results obtained by using the proposed active contour driven by $\mathrm{CNN}$ for spot segmentation (see Figure 4).

Further on, the reproducibility and biological significance of the proposed image processing techniques are assessed in the case of the presented dataset. The mean absolute error (MAE) and the coefficient of variation $(\mathrm{CV})$, which indicate the sameness of spot intensities and the variation of spot intensities, respectively, are computed to quantify the reproducibility of the proposed segmentation techniques.

The MAE is given by:

$$
M A E_{\text {spot }} \frac{1}{n} \sum_{i=1}^{n}\left|G_{i}^{n}-\bar{G}\right|
$$

where $\mathrm{n}$ is the number of replicates, $G_{i}{ }^{n}$ is the normalized mean spot intensity value and $\bar{G}$ is the spot overall mean if one considers the means of the corresponding spots within the $\mathrm{n}$ replicates.

The CV is given by:

$$
C V_{\text {spot }}=\frac{\sigma}{\mu}
$$

where $\sigma$ and $\mu$ represent the standard deviation of spot intensity with subtracted background and the mean spot intensity, respectively.

The efficiency of the proposed method increases as the MAE and CV values decrease. Both the proposed segmentation method and the GenePix Pro Software approach had MAE and CV values computed. As it is shown in Figure 5, the following parameter values were obtained for the E1 experiment: the average $M A E_{A C M-C N N}$ coefficient was 518 and the average $M A E_{G P}$ measured by GenePix Pro software was 551. Furthermore, the MAEACM-CNN, and MAEGP average values for experiment E2 were 541 and 495, respectively. In terms of MAE and CV coefficient, the level-set segmentation protocol produces results which are comparable to those of the GenePix Pro method. In the case of spot pixel intensity values, the $\mathrm{CV}$ represents a standardized metric of dispersion that is independent of the unit where the measurement was taken. A small $\mathrm{CV}$ shows that the pixel intensity values for a given microarray spot differ slightly. As a consequence, considering the $\mathrm{CV}$ coefficients, Table 1 shows that the proposed approach delivers a more accurate spot description.
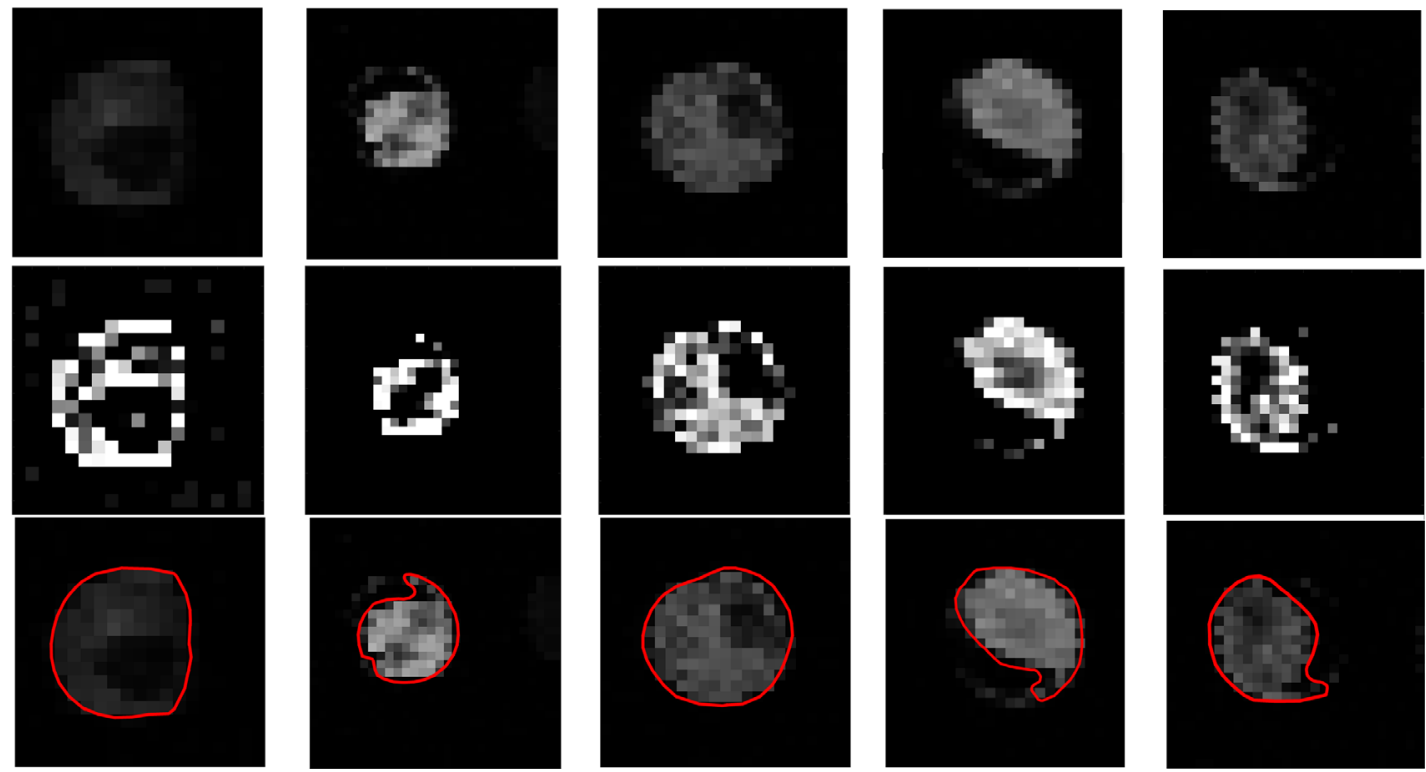

Figure 4. (i) the first row ilustrates the microarray spots with irregular contours selected for segmentation, having the following identification numbers according to GenePix Experiment GSM33353: IDs 537, 9458, 9454, 2583 and 10565; (ii) the second row ilustrates the edge features determined using the CNN approach; (iii) the third row shows the results of the proposed spot segmentation procedure 
The genes differentially expressed within a microarray experiment are estimated once the intensity values for each microarray spot are measured, and they are known as up-/downregulated genes. The interpretation of relative variations in intensities for the same spot from the sample and reference images, namely $I_{C y 3}$ and $I_{C y 5}$, respectively, is of interest to biologists and medical doctors. The fold change value $F_{C}=\log 2(R / G)$ is employed for selecting he differentially expressed genes, where $\mathrm{R}$ and $G$ represent the median intensity for the same microarray spot recorded from $I_{C y 3}$ and $I_{C y 5}$ fluorescent label, respectively. As a rule of thumb, a fold change value $F_{C}>1.5$ marks a gene corresponding to a microarray spot as upregulated or over-expressed, which means that the gene is active in the case of the microarray experiment under analysis.

Table 1. Comparison of the values of the coefficient of variation $(\mathrm{CV})$ determined based on the proposed segmentation procedure ( second column) and the ones delivered by GenePix Pro

\begin{tabular}{|c|c|c|}
\hline $\begin{array}{c}\text { Experiment ID } \\
\text { (Image channel) }\end{array}$ & $\begin{array}{c}\text { Mean } \boldsymbol{C V} \\
\text { (proposed } \\
\text { method) }\end{array}$ & $\begin{array}{c}\text { Mean } \boldsymbol{C V} \\
\text { (GenePix) }\end{array}$ \\
\hline GSM333336 $\left(I_{C y 3}\right)$ & 0.586 & 0.663 \\
\hline GSM333336 $\left(I_{C y 5}\right)$ & 0.496 & 0.534 \\
\hline GSM333353 $\left(I_{C y 3}\right)$ & 0.439 & 0.635 \\
\hline GSM333353 $\left(I_{C y 5}\right)$ & 0.648 & 0.706 \\
\hline GSM333337 $\left(I_{C y 3}\right)$ & 0.592 & 0.684 \\
\hline GSM333337 $\left(I_{C V 5}\right)$ & 0.543 & 0.766 \\
\hline GSM333341 $\left(I_{C y 3}\right)$ & 0.472 & 0.552 \\
\hline GSM333341 $\left(I_{C V y}\right)$ & 0.622 & 0.795 \\
\hline
\end{tabular}

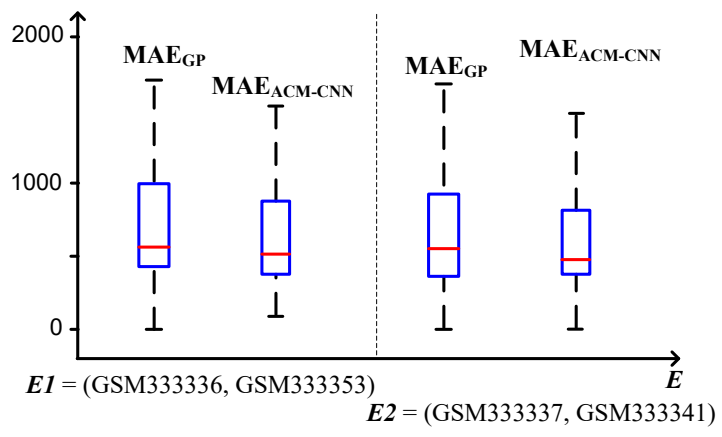

Figure 5. The reproducibility of the proposed spot segmentation approach indicated by the sameness of the spots evaluated using the mean absolute error $\left(\mathrm{MAE}_{\mathrm{ACM}-\mathrm{CNN}}\right)$ on the ordinate axis in case of the two sets of microarray image samples E1 and E2

Considering the proposed selection of spots, the fold change factor for the spots having the IDs
2583 and 9458 shows an increase in the $F c$ by 0.6 and 0.52 in comparison with GenePix Pro, which obtained the values of 1.62 and 1.54 .

This shows an activation of the At5g38430 gene (index 2583) involved in the process of converting the carbon dioxide into energy-rich molecules, whereas the AT3G09440 gene (index 9458) is part of a family of heat-shock proteins produced by plant cells in response to stressful conditions.

\subsection{Microfluidic Device Images}

It is well-known that gene expression (i.e. the translation of information encoded in a gene into complex molecules such as proteins which play critical roles in a living organism) is involved in the development and maintenance of multicellular organisms (Venkataramana et al., 2018). Any alteration of these processes may have a critical impact on the evolution of an organism such as cell motility that may lead to primary tumor formation. Consequently, along with the gene expression analysis, the cell and cell clusters have to be characterized also in terms of their physical features.

Some important tools for cell features extraction are the microfluidic devices. The manipulation of fluids that are geometrically confined to a submillimetre scale is known as microfluidics. These microfluidic system constraints include benefits such as cost-effectiveness and a high resolution, as well as features such as laminar flow in the case of both single and cluster cells navigating through a complex area (Hong et al., 2016; Umer et al., 2018). Microfluidics systems with several channels are constructed with these advantages in mind, and cultured cells are connected to the channel surface. This lays the groundwork for a cell cluster to enter the channels for further analysis. Mathematical models are available for describing the motion of a viscous fluid in porous media, in our case the microfluidic channels (Gutt, 2020). Cells and cell clusters contained in the microfluidic devices are moving through the microfluidic channels based on the gradient of nutrients using cell adhesion molecules. Thus, complex mathematical tools are available for modeling and evaluating cell cluster motility. Nevertheless, this paper is focused on the segmentation of microfluidics images for the determination of cells and cell clusters. 
A mention regarding the size of the clusters of cells found in the blood or lymphatic circulatory system of patients with several metastatic carcinomas is given in (Hong et al., 2016). They can range in size from 20 to $130 \mu \mathrm{m}$ and contain up to 100 cells, with the metastatic potential of up to $50 \%$ higher than that of single cells (Umer et al., 2018; Lisencu et al., 2021). Significant advances have been reported regarding the molecular analysis of tumor clusters, nevertheless, the limitation of state-ofthe-art research with regard to the cluster cells dynamics process lies in the scarce information regarding the assessment of cell cluster velocity in relation to various factors such as cluster size or events (i.e., cluster division or unions). Thus, understanding the key phenotypes of cell clusters is an open subject in current research. Using microfluidic devices, images of cluster cells along the microfluidic channels are recorded (Au et al., 2017). State-of-the-art research shows an increased interest in the analysis of the cell clusters migration process (Spatarelu et al., 2019; Ma et al., 2018). Collective migration in the case of single cells was analyzed, showing that it is associated with biomechanical particularities such as the restructuring of extracellular matrix (ECM) (Spatarelu et al., 2019). Considering cell clusters, the use of microfluidic devices with narrow constrictions for the channels (i.e. channel width of 5-10 $\mu \mathrm{m}$ ) proved that the process of extravasation in the case of cell clusters occurs more often than in the case of single cells (i.e. in $90 \%$ of clusters, up to 20 cells were found to migrate through the narrowest constriction of $5 \mu \mathrm{m}$ ) (Ma et al., 2018). Nevertheless, more information is necessary regarding cell clusters that are not confined to a constricted area. Thus, the questions that arise are: What is the cell clusters size in the case of microfluidic channels having a width of $50 \mu \mathrm{m}$, with the possibility to host cell clusters of large size (in round numbers up to 20 - 30 cells per cell cluster (Umer et al., 2018))? Are the cell clusters formed even in case that the channel size is not limited? The following dataset and the proposed method are used further on to answer the previous questions.

\subsection{Dataset, Results and Discussion}

In the case of the proposed dataset, the microfluidic channels were designed to have a channel width of $50 \mu \mathrm{m}$, in order to allow cell clusters of various sizes to be present within the same channel. For obtaining the cell cluster images, transfected suspension cells were loaded into the seeding chamber of collagen-coated microfluidic devices and allowed to migrate for at least 24 hours (Tudoran et al., 2015). Migrating cells were recorded using a Nikon BioStation microscope. Time-lapse images were acquired at multiple spatial locations for a number of 10 microfluidic experiments, leading to a dataset of over 30 images that were considered for cell cluster segmentation using the proposed approach. Each image recording of 16 microfluidic channels with 0 to 3 cell clusters within each channel led to a number of over 500 cell clusters available for analysis. Microfluidic channels hosting mammary cancer cell lines are ilustrated in Figure 6.

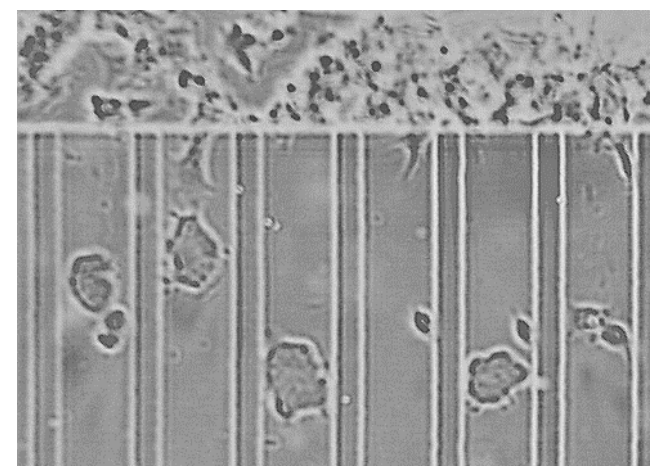

Figure 6. Microfluidic channels hosting mammary cancer cell lines

Using the proposed segmentation approach, a rectangular perimeter using active contour models guided by the CNN edge features is evolved as described by equation (6). At the end of the curve evolution process, the resulted separates the pixels corresponding to the cells or cell clusters from the background. The image horizontal profile was computed as the preliminary procedure for the determination of the initial rectangular perimeter which was used as the initial condition for the curve evolution. The indices for the maximum values within the image profile correspond to the set $H_{i}$ of horizontal lines $h_{i}$, whereas every two consecutive lines hi and $h_{i}+1$ delineate a given microfluidic channel $i$. Based on the values of the pairs $\left(h_{i}, h_{i+1}\right)$ the rectangular perimeter is defined. The curve evolution is applied separately on subimages corresponding to each of the microfluidic channels, as it is illustrated in Figures 7 and 8. As preprocessing steps, on each of the sub-images corresponding to the microfluidic channels, a padding procedure which replicates the image

https://www.sic.ici.ro 
margins followed by a Gaussian convolution using a convolution kernel of size 5 and $\sigma=1$ are applied.
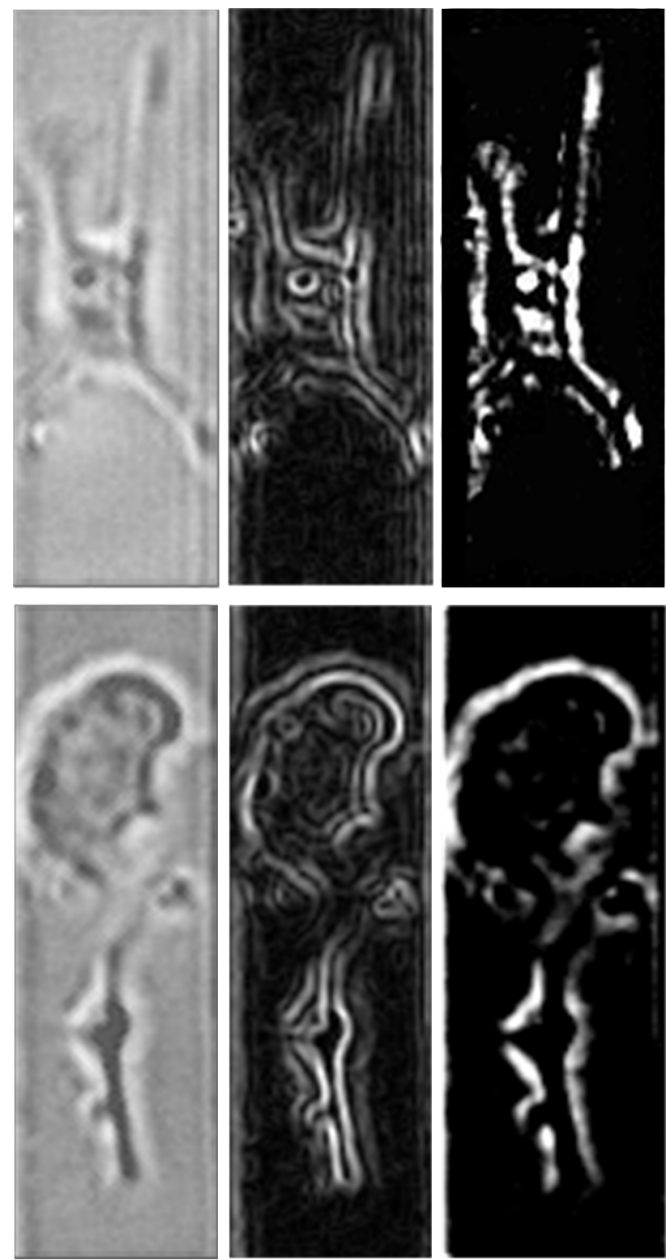

Figure 7. Visual results for two selected cell clusters (first column of images) together with the representation of the image gradient and CNN edge features in the second and third column of images, respectively
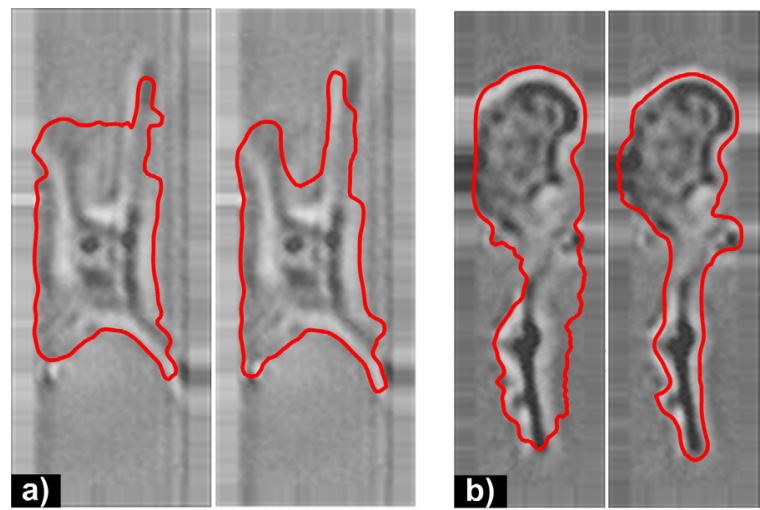

Figure 8. Segmentation of cell clusters during: (a) their movement using both the classic active contour approach (left) and the active contours based on CNN edge features (right) and (b) events such as cluster division or union using both the classic active contour (left) and the CNN driven active contour (right)
At this point, the initial curve is evolved by using both the classic active contour model and active contour models driven by edge features determined using $\mathrm{CNN}$.

The main benefits of the last approach are illustrated in Figure 8, in comparison with a classic active contour model. In the case of cell adhesion molecules attached to the surface of the microfluidic channel and used for the cell or cell clusters to advance, better visual results are obtained by using the proposed method. In Figure 8 (a) the second image shows that cell-specific adhesion molecules are detected more accurately. Regarding the weakly expressed edges, Figure 8(b) shows a comparison between the classic active contours and the active contours based on $\mathrm{CNN}$. Here, in the case of the cell cluster edges from the right side, the proposed approach also delivers more accurate segmentation results.

The cells within all channels are analyzed in terms of their shape and size using the proposed approach for segmentation. The gradient and edge features determined for the cell clusters, are also illustrated in Figure 7. Thus, in Figure 7 first line of images is described as a moving cell cluster, where the cell-specific phenotype is visible and used for the migration of the cell. In the second line of images, the cell cluster has an unusual shape, which is due to the cell cluster division process which may take place. The proposed segmentation procedure is tested in both cases. Referring to Figure 7, the second image column reveals the gradient magnitude in the case of cell cluster images, whereas the third image column shows the edge features determined by using the cellular neural network.

In terms of cell cluster sizes, both single-cell and cell clusters sizes were determined for the entire dataset, leading to an average size of $520 \mu \mathrm{m}$ for the single cells. Moreover, the size ranges between 700 to $1200 \mu \mathrm{m}$ for 29.6 percent and between 1200 and $5000 \mu \mathrm{m}$ for 60.9 percent of the entire cell cluster population. It can be noticed that only 9.5 percent of it corresponds to singlecells, showing that, even in conditions of nonextravasation (no channel constriction imposed), there is a preference for the tumor cells to migrate in large clusters. 
The accurate estimation of gene expression levels is also important in describing phenotypes for living organisms. Considering the aforementioned key aspects, the main novelties this paper brought about are the proposed segmentation procedure for accurate determination of cell cluster sizes using microfluidics devices and the determination of supplementary differentially expressed genes in comparison with the existing software so as to better explain the key biological processes involved in plant defense responses to pathogen infection.

\section{Conclusion}

Two of the major research goals in this field of biomedical applications are the use of microarray technology for an accurate description of genetic mechanisms governing different bio-molecular pathways involved in different diseases (Berry et al., 2019) together with the characterization of cluster cell phenotypes which have a leading role in tumor migration and metastasis (Micalizzi et al., 2017). The present paper proposes a segmentation procedure used in the computation of microarray spot average intensities and the determination of the features of cells and cell clusters. The employed procedure showed increased segmentation accuracy in case of image objects with weakly expressed edges in comparison with classic active contour models. Also, a more accurate identification of cellspecific motion parts has been observed (i.e., organelles). These results show great potential in both the characterization and the identification of events such as cluster cell division or merging events for the cell migration process. Moreover, in the case of microarray spot segmentation, the proposed procedure led to the identification of supplementary up-regulated genes in comparison with the number of up-regulated ones determined with the existing software platforms (i.e., GenePix Pro). The proposed CNN-driven active contours model is also employed for the analysis of the cell cluster migration process. It was found that tumor cells prefer to migrate in clusters in nonconstrictive microfluidic channels.

\section{Acknowledgments}

This work was supported through a grant from the Romanian Ministry of Education and Research, CNCS - UEFISCDI, project number PN-III-P4ID-PCE-2020-0368, within PNCDI (National Plan for Research, Development and Innovation) III.

\section{REFERENCES}

Au, S., Edd, J., Stoddard, A. E., Wong, K. H., Fachin, F., Maheswaran, S., Haber, D., Stott, S., Kapur, R. \& Toner, M. (2017). Microfluidic Isolation of Circulating Tumor Cell Clusters by Size and Asymmetry, Scientific Reports, 7(1), 2433.

Barburiceanu, S., Terebes, R. \& Meza, S. (2021). 3D Texture Feature Extraction and Classification Using GLCM and LBP-Based Descriptors, Applied Sciences, 11(5), 2332.

Belean, B., Borda, M., Ackermann, J., Koch, I. \& Bălăcescu, O. (2015). Unsupervised image segmentation for microarray spots with irregular contours and inner holes, BMC Bioinformatics, 16,412 .

Belean, B., Gutt, R., Costea, C. \& Balacescu, O. (2020). Microarray image analysis: from image processing methods to gene expression levels estimation, IEEE Access, 8, 159196 - 159205.
Berry, N. K., Scott, R. J., Rowlings, P. \& Enjeti, A. K. (2019). Clinical use of SNP-microarrays for the detection of genome-wide changes in haematological malignancies, Critical Reviews in Oncology / Hematology, 142, 58-67.

Campbell, A. M., Hatfield, W. T. \& Heyer, L. (2007). Make microarray data with known ratios, $C B E$ - Life Sciences Education, 6, 196-197.

Chua, L. O. \& Yang, L. (1988). Cellular Neural Networks: theory, IEEE Transactions on Circuits and Systems, 35(10), 1257-1272.

Daniels, C. \& Gallagher, F. (2018). Unsupervised Segmentation of 5D Hyperpolarized Carbon-13 MRI Data Using a Fuzzy Markov Random Field Model, IEEE Transactions on Medical Imaging, 37(4), $840-850$.

Elango, P. \& Murugesan, K. (2009). Digital Image Inpainting Using Cellular Neural Network,

https://www.sic.ici.ro 
International Journal of Open Problems in Computer Science and Mathematics, 2(3), 439-450.

Fabro, G., Di Rienzo, J. A., Voigt, C., Savchenko, T., Dehesh, K., Somerville, S. \& Alvarez, M. (2008). Genome-wide expression profiling Arabidopsis at the stage of Golovinomyces cichoracearum haustorium formation, Plant Physiology, 146(3), 1421-1439.

Gavrea, R., Bolinger, A., Pop, V., Isnard, O., Coldea, M. \& Benea, D. (2017). Influence of Cu Doping on the Electronic Structure and Magnetic Properties of the Mn2VAl Heusler Compound, Physica Status Solidi B - Basic Solid State Physics, 254(11), 1700160.

Gutt, R. (2020). Boundary Element Method for the Lid-driven Problem related to the Darcy-ForchheimerBrinkman System, Journal of Porous Media, 23(8), 821-835.

Hagerty, J., Stanley, R., Almubarak, H. A., Lama, N., Kasmi, R., Guo, P., Drugge, R., Rabinovitz, H., Oliviero, M. \& Stoecker, W. (2019). Deep Learning and Handcrafted Method Fusion: Higher Diagnostic Accuracy for Melanoma Dermoscopy Images, IEEE Journal of Biomedical and Health Informatics, 23(4), 1385-1391.

Han, B., Zhang, L. \& Gao, X. T. (2018). The region based MMTD energy function for image segmentation, Multimedia Tools and Applications, 78, 16695-16726.

Hong, Y., Fang, F. \& Zhang, Q. (2016). Circulating tumor cell clusters: What we know and what we expect (Review), International Journal of Oncology, 49(6), 2206-2216.

Hrošik, R. C., Tuba, E., Dolicanin, E., Jovanovic, R. \& Tuba, M. (2019). Brain Image Segmentation Based on Firefly Algorithm Combined with K-means Clustering, Studies in Informatics and Control, 28(2), 167-176. DOI: $10.24846 / v 28 \mathrm{i} 2 \mathrm{y} 201905$

Jiao, X., Chen, Y. \& Dong, R. (2020). An unsupervised image segmentation method combining graph clustering and high-level feature representation, Neurocomputing, 409, 83-92.

Li, C., Chen, X., Gui, C. \& Fox, M. (2010). Distance Regularized Level Set Evolution and Its Application to Image Segmentation, IEEE Transactions on Image Processing, 19(12), 3243-3254.

Li, X., Liao, X., Li, C., Huang, H. \& Li, C. (2011). Edge detection of noisy images based on cellular neural networks, Communications in Nonlinear Science and Numerical Simulation, 16(9), 3746-3759.

Lisencu, L. A., Bonci, E.-A., Irimie, A., Balacescu, O. \& Lisencu, C. (2021). The Role of Circulating Tumor Cells in Chemoresistant Metastatic Breast Cancer, Journal of Clinical Medicine, 10(4), 684.
Ma, Y., Middleton, K., You, L. \& Sun, Y. (2018). A review of microfluidic approaches for investigating cancer extravasation during metastasis, Microsystems \& Nanoengineering, 4, 1-13.

Micalizzi, D., Maheswaran, S. \& Haber, D. (2017). A conduit to metastasis: circulating tumor cell biology, Genes \& Development, 31(18), 1827 - 1840.

Nossek, J. A. (1996). Design and Learning with Cellular Neural Networks, International Journal of Circuit Theory and Applications, 24, 15-24.

Pal, N. R. \& Pal, S. K. (1993). A review on image segmentation techniques, Pattern Recognition, 26(9), $1277-1294$

Prasad, P. M. K., Prasad, D. Y. V. \& Sasibhushana Rao, G. (2016). Performance Analysis of Orthogonal and Biorthogonal Wavelets for Edge Detection of X-ray Images, Procedia Computer Science, 87, 116-121.

Rahmat, R. \& Birtill, D. B. (2018). Comparison of level set models in image segmentation, IET Image Processing, 12, 2212-2221.

Roska, T. \& Vandewalle, J. (1993). Cellular Neural Networks. John Wiley \& Sons.

Spatarelu, C. P., Zhang, H., Nguyen, D. T., Han, X., Liu, R., Guo, Q., Notbohm, J., Fan, J., Liu, L. \& Chen, Z. (2019). Biomechanics of Collective Cell Migration in Cancer Progression: Experimental and Computational Methods, ACS Biomaterials Science \& Engineering, 5(8), 3766-3787.

Su, T.-J., Wei, C.-P., Huang, S.-C. \& Hou, C.-L. (2008). Image noise cancellation using linear matrix inequality and cellular neural network, Optics Communications, 281(23), 5706-5712.

Terebes, R., Malutan, R, Borda, M, Germain, C., Bombrun, L. \& Ilea, I. (2018). Polarimetric Radar Image Classification Using Directional Diffusion And Descriptive Statistics, Revue Roumaine des Sciences Techniques - Serie Electrotechnique et Energetique, 63(1), 83-88.

Tudoran, O., Virtic, O., Bălăcescu, L., Lisencu, C., Fetica, B., Gherman, C., Bălăcescu, O. \& BerindanNeagoe, I. (2015). Baseline blood immunological profiling differentiates between Her2-breast cancer molecular subtypes: Implications for immunomediated mechanisms of treatment response, OncoTargets and Therapy, 8, 3415-3423.

Umer, M., Vaidyanathan, R., Nguyen, N. T. \& Shiddiky, M. J. A. (2018). Circulating tumor microemboli: Progress in molecular understanding and enrichment technologies, Biotechnology Advances, 36(4), $1367-1389$ 
Venkataramana, L., Jacob, S. G. \& Ramadoss, R. (2018). Parallelized Classification of Cancer Subtypes from Gene Expression Profiles Using Recursive Gene Selection, Studies in Informatics and Control, 27(2), 213-222. DOI: 10.24846/v27i2y201809

Vese, L. A. \& Guyader, C. (2015). Variational Methods in Image Processing, Mathematical and Computational Imaging Sciences Series. Taylor \& Francis.

Vrejoiu, M. H. (2020). License Plate Segmentation in Images Based on per-Block Contrast Analysis and CCA, Studies in Informatics and Control, 29(2), 189203. DOI: $10.24846 / \mathrm{v} 29 \mathrm{i} 2 \mathrm{y} 202005$
Wang, X. \& Huang, D. (2009). A novel density-based clustering framework by using level set method, IEEE Transactions on Knowledge and Data Engineering, 21(11), 1515-1531.

Xing, F., Xie, Y., Su, H., Liu, F. \& Yang, L. (2018). Deep Learning in Microscopy Image Analysis: A Survey, IEEE Transactions on Neural Networks and Learning Systems, 29(10), 4550-4568.

Zou, L., Song, L.-T., Weise, T., Wang, X.-F., Huang, Q.-J., Deng, R. \& Wu, Z.-Z. (2021). A survey on regional level set image segmentation models based on the energy functional similarity measure, Neurocomputing, 452(19), 606-622. 